\title{
Folic Acid Enhances Early Functional Recovery in a Piglet Model of Pediatric Head Injury
}

\author{
Maryam Y. Naim ${ }^{a}$ Stuart Friess ${ }^{a}$ Colin Smith $^{c} \quad$ Jill Ralston ${ }^{b}$ Karen Ryall ${ }^{b}$ \\ Mark A. Helfaer ${ }^{a}$ Susan S. Margulies ${ }^{b}$ \\ aDepartment of Anesthesiology and Critical Care Medicine, Children's Hospital of Philadelphia, and \\ ${ }^{b}$ Department of Bioengineering, University of Pennsylvania, Philadelphia, Pa., USA; ' ${ }^{c}$ Department of \\ Neuropathology, Western General Hospital, Edinburgh, UK
}

\section{Key Words}

Neuroprotection • Folic acid • Traumatic brain injury • Neurobehavioral assessment $\cdot$ Pediatric brain injury • Axonal injury · Swine

\begin{abstract}
For stroke and spinal cord injury, folic acid supplementation has been shown to enhance neurodevelopment and to provide neuroprotection. We hypothesized that folic acid would reduce brain injury and improve neurological outcome in a neonatal piglet model of traumatic brain injury (TBI), using 4 experimental groups of 3- to 5-day-old female piglets. Two groups were intubated, anesthetized and had moderate brain injury induced by rapid axial head rotation without impact. One group of injured (Inj) animals received folic acid (Fol; $80 \mu \mathrm{g} / \mathrm{kg}$ ) by intraperitoneal (IP) injection $15 \mathrm{~min}$ following injury, and then daily for 6 days $(I n j+F o l ; n=7)$. The second group of injured animals received an IP injection of saline (Sal) at the same time points (Inj + Sal; $\mathrm{n}=8$ ). Two uninjured (Uninj) control groups (Uninj + Fol, $\mathrm{n}=8$; Uninj + Sal, $\mathrm{n}=7$ ) were intubated, anesthetized and received folic acid $(80 \mu \mathrm{g} / \mathrm{kg})$ or saline by IP injection at the same time points
\end{abstract}

as the injured animals following a sham procedure. Animals underwent neurobehavioral and cognitive testing on days 1 and 4 following injury to assess behavior, memory, learning and problem solving. Serum folic acid and homocysteine levels were collected prior to injury and again before euthanasia. The piglets were euthanized 6 days following injury, and their brains were perfusion fixed for histological analysis. Folic acid levels were significantly higher in both Fol groups on day 6 . Homocysteine levels were not affected by treatment. On day 1 following injury, the Inj + Fol group showed significantly more exploratory interest, and better motor function, learning and problem solving compared to the Inj + Sal group. Inj + Fol animals had a significantly lower cognitive composite dysfunction score compared to all other groups on day 1 . These functional improvements were not seen on day 4 following injury. Axonal injury measured by $\beta$-amyloid precursor protein staining 6 days after injury was not affected by treatment. These results suggest that folic acid may enhance early functional recovery in this piglet model of pediatric head injury. This is the first study to describe the application of complex functional testing to assess an intervention outcome in a swine model of TBI.

Copyright $\odot 2011$ S. Karger AG, Basel

\section{KARGER}

(C) 2011 S. Karger AG, Basel

Fax +4161306 1234

E-Mail karger@karger.ch

www.karger.com
Accessible online at: www.karger.com/dne
Susan Margulies, $\mathrm{PhD}$

Department of Bioengineering, University of Pennsylvania

240 Skirkanich Hall, 210 South 33rd Street

Philadelphia, PA 19104-6321 (USA)

Tel. +1 215898 0882, Fax +1 215573 3808,E-Mail margulies@ seas.upenn.edu 


\section{Introduction}

Traumatic brain injury (TBI) is the most common cause of death among children in the USA with an annual incidence of 63 hospitalizations and 4.5 deaths per 100,000 children aged $0-14$ years [1]. TBI involves a primary injury that results from distortion of the brain parenchyma, and secondary injury attributed to cell death cascades, excitotoxicity, secondary cerebral swelling, traumatic axonal injury (TAI) and inflammation [2]. TAI contributes to the morbidity after TBI [3] and appears to be prevalent in victims of pediatric abusive head trauma [4]. Classically, the primary mechanism of TAI was parenchymal shearing at the time of primary injury. More recently, TAI has been shown to occur frequently by a delayed process termed 'secondary axotomy'. The current TAI paradigm is that mechanical damage progresses from an initial ionic derangement when the permeability barrier provided by the plasmalemma is breached, to ion influx resulting in necrotic or apoptotic cell death and impairment of axoplasmic flow secondary to a direct cytoskeletal abnormality [5].

The importance of folic acid in central nervous system development was established by the observation that folic acid-deficient, pregnant women had a greatly increased risk of neural tube defects in their babies [6], which was eliminated by periconceptional folic acid supplementation [7]. Moreover, inborn errors of folic acid transport and metabolism in childhood are associated with developmental delay, motor and gait abnormalities, and seizures [8]. In adults, folic acid deficiency contributes to dementia, impaired cognition, depression, psychosis and Alzheimer's disease, and can cause subacute combined degeneration of the spinal cord $[9,10]$. In a stroke model with wild-type mice, folic acid deficiency was associated with increased oxidative DNA damage and larger ischemic injury volume, augmenting brain damage after middle cerebral artery occlusion and reperfusion [11]. In recent studies, neonates with higher folic acid levels had better vascular endothelial function than those with lower levels [12].

As a neuroprotectant, folic acid supplementation reduces the incidence of hemorrhagic stroke in adults [13]. Folic acid also enhances repair processes in an adult rat model of spinal cord injury, with administration of folic acid prior to and following injury improving functional recovery [14]. The pluripotential protective effect of folic acid is hypothesized to be that folate is a cofactor in the remethylation of homocysteine, which reduces homocysteine accumulation, a cytotoxic amino acid that can in- duce DNA strand breakage, oxidative stress and apoptosis [15].

There is no proven targeted therapy for TBI, and treatment remains largely supportive. Recently, in cultured mouse cerebellar granule neurons, folic acid was shown to ameliorate glutamate and $N$-methyl-D-aspartate (NMDA)-induced neuronal death in a time- and dosedependent manner, demonstrating that folic acid may have therapeutic potential in addition to prevention of brain injury [16].

Based on these new data using folic acid in an in vitro TBI model - and the established role for folic acid in central nervous system development, neurodegenerative disorders and stroke -, we hypothesized that folic acid supplementation after injury would decrease the severity of TAI in our well-established piglet model of moderate pediatric head injury $[17,18]$. An inertial rotation device was used to create diffuse brain injury in 3- to 5-day-old piglets to represent the human infant at less than 3 months of age. Neurological outcome was assessed using instruments developed in our laboratory for behavioral, cognitive and motor function assessment in piglets $[18,19]$.

\section{Methods}

\section{Experimental Design}

The institute of animal care and use committee of the University of Pennsylvania approved all protocols. Neonatal piglets (3-5 days old; females: $\mathrm{n}=40$; males: $\mathrm{n}=10$ ) were studied in 10 groups consisting of 5 littermates per group. Two days prior to injury, the animals were acclimated to the research staff for $1 \mathrm{~h}$ each day, and were trained on how to cross a wide beam. On the day on injury, of each group, 1 animal was injured (Inj) and received folic acid (Fol) by intraperitoneal (IP) injection immediately after injury (Inj + Fol), and daily thereafter. One animal was injured and received IP saline (Sal; Inj + Sal). Of the uninjured (Uninj) controls, 1 received folic acid (Uninj + Fol) and 1 received IP saline (Uninj + Sal). A fifth piglet from each litter (male; Pig-X) served as a con stimulus for behavioral testing. Behavioral testing occurred on days 1 and 4 following injury. These days were selected based on previous studies from our laboratory where testing occurred on days 1, 4, 6, 8 and 11 following injury and the greatest differences in behavior between injured and sham animals were seen on days 1 and 4 following injury [19]. The doses of folic acid and normal saline were repeated every day for the period of the study. On day 6 , the piglets were anesthetized, had blood drawn, were euthanized, and their brains were perfusion fixed with formalin for histological analysis (fig. 1).

\section{Preinjury Preparation}

The animals were anesthetized via snout mask with isoflurane $4 \%$, intubated and maintained on $100 \%$ oxygen while breathing spontaneously. Ventilation support was provided as necessary. The proper plane of anesthesia was defined as absence of with- 
Fig. 1. Time line of study. Piglets underwent 2 days of acclimation to the research staff, followed by injury, behavioral testing on days 1 and 4 following injury, and daily folic acid injections following injury. The animals were euthanized on day 6 .

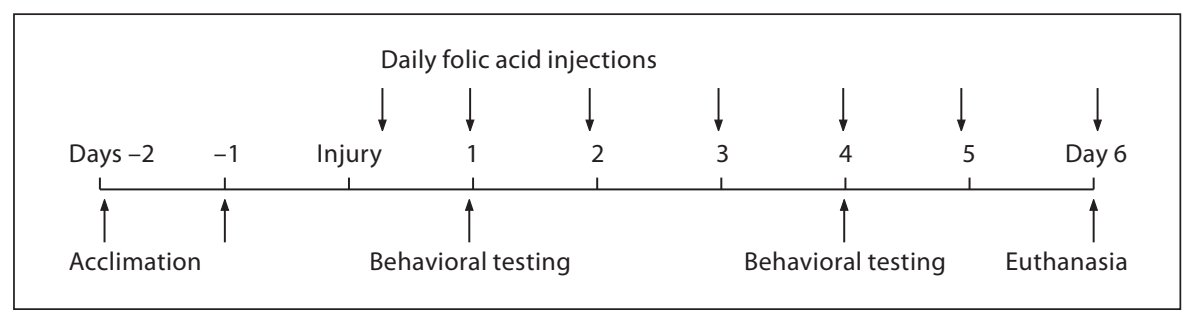

drawal to pinch. Noninvasive blood pressure (blood pressure cuff on hind limb), oxygen saturations (pulse oximetry probe, MDE Escort II; MDE, Ariera, Calif., USA), core body temperature (rectal thermometer probe) and end-tidal carbon dioxide (VetCap model 2050081; SDI, Waukesha, Wisc., USA) were monitored during the injury. Each animal had $2 \mathrm{ml}$ of blood drawn to measure levels of folic acid and homocysteine. Each animal received analgesics (meloxicam $0.2 \mathrm{mg} / \mathrm{kg}$ i.m. in the initial 2 groups; buprenorphine $0.02 \mathrm{mg} / \mathrm{kg}$ i.m. in 8 groups; see Results section) prior to the injury procedure. While maintained on $1-2 \%$ isoflurane, the animals' heads were secured to the inertial injury apparatus by a padded snout clamp.

\section{Injury}

Head injury was induced by activation of the inertial loading device, the HYGE pneumatic actuator [17], which rapidly rotated the head of the animal through a 90 - to 110 -degree arc in the axial plane, with the center of rotation in the cervical spine. The animals were removed from the bite plate and respiratory support was provided as needed. Immediately following injury, $80 \mu \mathrm{g} / \mathrm{kg}$ of folic acid was administered by IP injection to the Inj + Fol animals. This dose was based on the dose that had the greatest effects on regeneration in the rat model of spinal cord injury [14]. Equivalent volumes of normal saline were administered by IP injection to 1 injured animal (Inj + Sal). Duration of unconsciousness was assessed by latency of return of pinch reflex, and vitals were monitored every 5-10 min. The animals were ventilated until return of spontaneous respiration. After return of pinch reflex and stable vital signs, the injured animals were extubated. They were observed continuously, and food and water were provided until they met the following criteria: vocalization (without squealing), steady ambulation, no aggression or avoidance behavior, no piloerection, and presence of proper feeding/drinking. After these criteria were met, the animals were returned to the animal housing facility.

\section{Sham Controls}

The sham animals underwent the same preinjury preparation as the injured animals and received buprenorphine for analgesia. While maintained on isoflurane, the animals' heads were secured on the biteplate, but they received no injury. Immediately following the sham injury, 1 animal received an IP injection of folic acid $($ Uninj + Fol), and 1 animal was injected with IP saline (Uninj + Sal). The animals received postinjury care as described for the injured animals above.

\section{Functional Testing Methods and Analysis}

A broad range of behaviors were evaluated on days 1 and 4 following injury to assess domains of cortical function relevant to human higher cortical functions that are compromised after TBI (fig. 1). All procedures involved operant-conditioning techniques with food as a positive reward, and no aversive conditioning was done. The battery of tests performed included exploratory interest evaluation by open-field testing and T-maze, visual-based problem solving by the glass barrier task, olfactory-based problem solving by the food cover task, motor function by balance beam, learning and antegrade memory by T-maze testing, social behavior by Pig-X, as previously described in detail $[18,19]$, and a new mirror test to evaluate visual exploration of new objects.

Each morning of testing, the animals were fasted for $2 \mathrm{~h}$ and weighed to monitor growth. The testing order was randomly assigned for the day. All tests were videotaped and transferred to a digital videodisk for scoring by an evaluator who was masked to group identification. After having completed a testing session, the animals were returned to the animal housing facility and allowed to feed ad libitum.

\section{Open Field}

Each animal was placed in an open field $(4 \times 8 \mathrm{ft})$ with various objects placed in predetermined locations. The behaviors (e.g. nudging toys, walking, sleeping) were scored as present/absent for every minute-long interval. The test space was divided into 9 imaginary zones, and the number of zones entered and lines crossed was measured.

After 10 min, the male littermate Pig-X was placed in the center of the open field for a 10 -min interval to assess social interaction and reaction of the test pig to a con-specific stimulus. The interpig distance and specific behaviors (sniffing, head butting, mounting, fighting and social sleeping) were scored for every minute-long interval.

\section{Mirror Test}

After having removed the objects from the open-field test, 2 new objects were positioned on the pen walls for a 10-min interval: a mirror $(12 \times 24 \mathrm{in})$, and a mirror reversed exposing its not reflective surface $(12 \times 24$ in). A third wall with no novel object was designated the 'null zone' (fig. 2). The test space was divided into 9 imaginary zones, and the time spent in front of the mirror, reversed mirror and null zones was tracked during 1-min intervals and reported as seconds per minute. Specific exploratory behaviors, including snout contact, pacing, backing up and approaching as well as nonsnout body contact between the piglet and mirror, reverse and null zone were also recorded. Each social behavior was scored as present or absent for each of the 1-minlong intervals, and reported as percent intervals that the behavior was observed. 


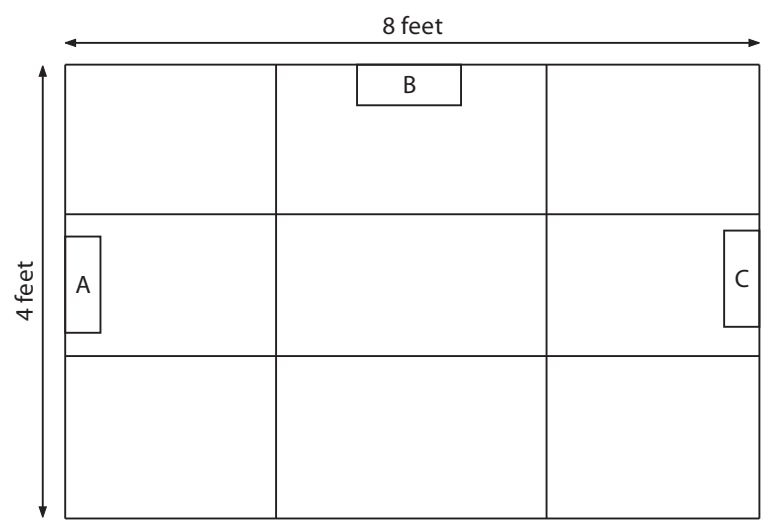

Fig. 2. Mirror test. After open-field testing, a mirror (A) was added to the test space, as well as a mirror reversed to expose its nonreflective surface (B). A third area, the null zone (C), was used to compare the frequency of the piglet in the zone of the mirror or reversed mirror. Time in seconds in the zones of the mirror, reversed mirror and null zone as well as specific behaviors were recorded per 1-min epoch for $10 \mathrm{~min}$.

\section{Glass Barrier Task}

In the glass barrier task, the piglets learned to walk around a transparent glass barrier to reach the food reward. The time to the food reward, the number of nudges at the glass barrier, the number of times the piglet walked away from the glass barrier, and the number of failures to reach the food were recorded for 5 consecutive trials.

\section{Food Cover Task}

In the food cover task, the piglets learned that an opaque cover consistently hid a food reward, and that by lifting the cover they gained access to the food. The time to food reward, the number of errors and the number of failures to reach food were recorded for 6 consecutive trials.

\section{Balance Beam}

Motor performance was tested by balance beam walking, utilizing a narrow beam with a food reward at the end. The time to complete the task, the number of failures, and the number of foot errors were recorded for 6 consecutive trials.

\section{T-Maze Test}

The T-maze consisted of 2 arms, with food bowls in each arm, which the animal only saw once fully committed to that arm of the maze. A bowl of milk replacer, not visible to the animal, was placed behind each arm to eliminate smell as a confounder. During the training phase, the animals were shown the arm of the Tmaze with no food reward, and then the arm with the food reward, and were given 20 opportunities to find the food reward. Success was defined as finding the food reward in less than $15 \mathrm{~s}$ without entering the other arm. The piglets were required to com- plete 5 correct trials to proceed to further T-maze testing. In the intramaze challenge, a movable pie plate was placed in the arm of the T-maze with the food reward, and the latency to reach the food reward, the number of errors and the length of time in contact with the pie plate were measured. In normal trials, the pie plate was removed and the pigs had 5 tries to find the food reward in the arm where the training and intramaze challenge had occurred. In reversal trials, the food reward was switched to the other arm of the T-maze, 6 additional trials were performed, and the time to reach the food reward, the number of errors, the number of visits to the old food, and the time spent at the old food were recorded.

\section{Euthanasia}

On day 6, the animals were anesthetized via snout mask (isoflurane $4 \%$ ), intubated and maintained on $100 \%$ oxygen while breathing spontaneously. Each animal received analgesics (meloxicam $0.2 \mathrm{mg} / \mathrm{kg}$ in the first 2 groups; buprenorphine 0.02 $\mathrm{mg} / \mathrm{kg}$ i.m. in 8 groups). Each animal had a blood sample drawn to measure levels of folic acid and homocysteine, and had a microdialysis probe placed (on a trial basis). The piglets were euthanized with an overdose of intravenous sodium pentobarbital (150 $\mathrm{mg} / \mathrm{kg}$ ). A median sternotomy and transcardial perfusion with 1 liter of heparinized saline $(5,000$ units/l) were performed. The brains were perfusion fixed in situ with $10 \%$ neutral buffered formalin (3.5 liters; Sigma Chemical, St. Louis, Mo., USA) and removed.

\section{Pathology and Histological Methods}

The formalin-fixed brains were examined by a neuropathologist masked to group identification. Macroscopic examination was performed with documentation of focal pathology such as subdural and subarachnoid hemorrhages and surface contusions. The brains were sectioned into 16 serial 3 -mm-thick coronal blocks including the cerebrum, brain stem and high cervical spinal cord. The sections were examined for tissue tears, intracerebral hemorrhage and subarachnoid hemorrhage. Following routine processing, tissue was embedded in paraffin wax, and $6-\mu \mathrm{m}-$ thick sections were cut from each 3-mm block for microscopic examination. The sections were stained with HE or with the immunohistochemical markers for axonal injury $\beta$-amyloid precursor protein $(\beta-\mathrm{APP})$, and lightly counterstained with Meyer's hematoxylin. All fields in the slides were examined microscopically at a scanning power $(\times 5-10$ magnification). Specific fields were examined at $\times 20-40$ magnification).

Assessment focused on the degree and distribution of cellular (particularly neuronal) injury and white matter damage in the Inj + Fol and Inj + Sal groups with comparisons to Uninj + Sal. The HE-stained sections were examined to document established infarcts (changes in staining intensity) and ischemic neurons (cell shrinkage and eosinophilia). Axonal injury was assessed by disruption to axonal flow ( $\beta$-APP immunohistochemistry). Regions of axonal injury, of subarachnoid and parenchymal hemorrhage and of cell death were noted on digital photographs of the coronal blocks.

Total brain area was measured by tracing the brain area in images of each of the 16 sections (Image J; National Institutes of Health, Bethesda, Md., USA) and by summing the results from all slices. The periphery of each region of $\beta$-APP reactivity noted by the neuropathologist on these images was traced in each slice us- 
Fig. 3. Flow diagram of study animal selection. There were 7 deaths in the injured animal group, 3 animals were excluded from behavioral analysis. The final numbers of animals used in the behavioral analysis were 15 injured animals and 15 uninjured animals.

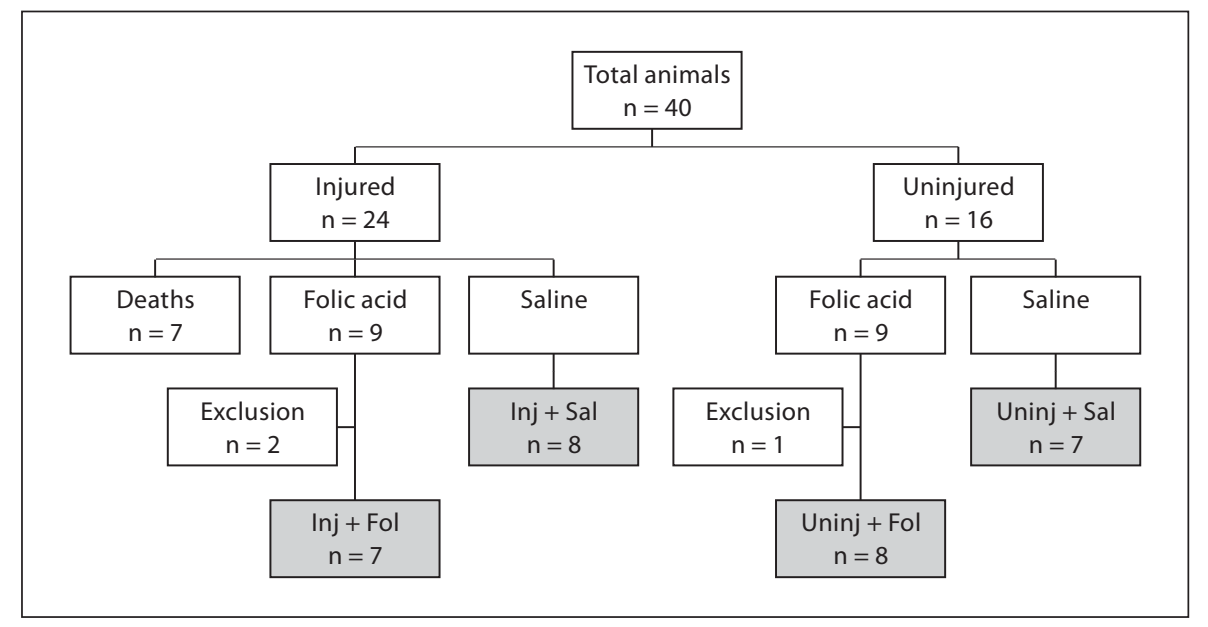

ing the same procedure, and summed to determine the total area of axonal injury in the entire brain. Fractional axonal injury area (in percent) was calculated by dividing the total area of injured white matter by total brain area. Similarly, ischemic brain injury was assessed, and the area of infarction (in percent) was calculated by dividing the total regional area of infarction by total brain area.

\section{Cognitive Composite Dysfunction Score}

The cognitive composite dysfunction (CCD) score is a measure of the overall neurobehavioral performance of the piglet. This score is based on the performance on tests where there are the most consistent responses among shams. Five measures were included in the calculation of this score: T-maze training failure rate; T-maze intramaze change time in contact with the novel object; latency to reach the food reward in the T-maze normal trials; latency to reach the food reward in the T-maze reversal trials, and percentage of time spent sniffing the wall of the test space in openfield testing [19]. For each of these 5 measurements, means and standard deviations (SD) from the means for the shams (Uninj + Sal) were calculated. Next, the performance of individual animals in each of the other groups (Inj + Fol, Inj + Sal, Uninj + Fol) on the selected behaviors were compared to the sham mean and SD. Each animal's z-score was calculated for each behavior by taking the difference between the individual animal's performance and the sham mean, and dividing it by the SD of the sham group. Scores for each of the 5 behaviors of an individual animal were summed to calculate the composite dysfunction. Next, the CCD was correlated with the percent white matter injury, as assessed by $\beta$-APP staining. In previous work in our laboratory, CCD correlated well with percent white matter injury [19].

\section{Statistical Analysis}

A reviewer masked to group identification scored the neurobehavioral tests. Comparisons were made between uninjured and injured groups, between treated and untreated groups, and between the 4 subgroups (Inj + Fol, Uninj + Fol, Inj + Sal and Uninj + Sal). For comparison of weights, unconsciousness times, folate and homocysteine levels, axonal injury, infarct volume, and comparison of CCD, the Student t test was used to compare the differences between injured and uninjured animals and between treated and untreated animals. Values of percent axonal injury and infarct volume were transformed into base-10 log for statistical analysis. Analysis of variance (ANOVA) testing was used for group comparisons in neurobehavioral testing. Two-way ANOVAs were used to assess group and day interactions for individual open-field behaviors, individual mirror behaviors, T-maze training and intramaze challenge. Typical degrees of freedom for these analyses were 1 when comparisons were made between treated and untreated groups and between injured and uninjured groups, and 3 when comparisons were made for all 4 subgroups. For those tests that involved trials (glass barrier, food cover, beam testing, T-maze normal and reversal trials) and scoring of behaviors during minute epochs (open-field, socialization and mirror testing), three-way ANOVAs were used to assess group, trial and day interactions, and group, minute epoch and day interactions. Typical degrees of freedom for these analyses were 1 when comparisons were made between treated and untreated groups and between injured and uninjured groups, 3 when comparisons were made for all 4 subgroups, and 12-15 when assessing subgroup trial and day interactions depending on whether there were 5 or 6 trials in a test. For group $\times$ minute interactions in the openfield and mirror tests, degrees of freedom were 9, and 27 for subgroup $\times$ minute interactions. Typical $\mathrm{f}$ values for significant behaviors ranged from 2.34 to 43.4. Statistical significance was defined as $p<0.05$. Post hoc group comparisons were examined using the Tukey-Kramer method with significance defined as $\mathrm{p}<$ 0.05 . All results are reported as mean \pm SD of mean, unless specified otherwise.

\section{Results}

\section{Mortality}

A total of 24 animals were injured (fig. 3), of which 7 animals were sacrificed within several hours following injury. Two of these 7 animals had hard palate fractures and were euthanized following injury. One animal be- 
Table 1. Summary of injury loads and clinical findings

\begin{tabular}{|c|c|c|c|c|c|c|}
\hline & $\begin{array}{l}\text { Angular velocity } \\
\mathrm{rad} / \mathrm{s}\end{array}$ & $\begin{array}{l}\text { Unconsciousness time } \\
\text { min }\end{array}$ & $\begin{array}{l}\text { Folic acid (day 6) } \\
\mathrm{ng} / \mathrm{ml}\end{array}$ & $\begin{array}{l}\text { Homocysteine (day 6) } \\
\mu \mathrm{mol} / \mathrm{l}\end{array}$ & $\begin{array}{l}\text { Preinjury weight } \\
\mathrm{kg}\end{array}$ & $\begin{array}{l}\text { Weight (day } 6 \text {, } \\
\mathrm{kg}\end{array}$ \\
\hline Inj + Fol & $193.29 \pm 5.31$ & $6.32 \pm 0.12^{\mathrm{a}}$ & $21.76 \pm 3.5^{1}$ & $6.73 \pm 0.8$ & $2.24 \pm 0.52$ & $3.12 \pm 0.87$ \\
\hline $\mathrm{Inj}+\mathrm{Sal}$ & $194.25 \pm 8.11$ & $6.23 \pm 0.09^{a}$ & $18.68 \pm 4.75$ & $6.88 \pm 1.72$ & $2.01 \pm 0.24$ & $2.95 \pm 0.39$ \\
\hline Uninj + Fol & & $4.55 \pm 0.1^{\mathrm{b}}$ & $24 \pm 0^{1}$ & $6.31 \pm 2.57$ & $1.8 \pm 0.54$ & $2.58 \pm 0.79$ \\
\hline Uninj + Sal & & $2.53 \pm 0.12$ & $18.08 \pm 4.84$ & $5.45 \pm 1.86$ & $1.99 \pm 0.39$ & $2.47 \pm 0.78$ \\
\hline
\end{tabular}

Values denote means $\pm \mathrm{SD}$. Angular velocities did not differ between injured groups. Durations of unconsciousness were significantly longer: ${ }^{\mathrm{a}} \mathrm{p}<0.05$, injured animals compared to uninjured animals; ${ }^{\mathrm{b}} \mathrm{p}<0.05$, uninjured animals that received folic acid compared to uninjured animals that received saline. There were no differences between piglet weights at baseline or on day 6 , and all groups gained weight over time.

${ }^{1}$ Groups that received folic acid had higher serum folic acid levels on day 6, but not lower homocysteine levels. came apneic and unresponsive following extubation, and had a cervical spine hematoma and pulmonary edema on necropsy. Three animals never regained consciousness following injury and had large subdural hematomas on necropsy that were attributed the use of the new preinjury analgesic meloxicam, a nonsteroidal anti-inflammatory agent with antiplatelet effects [20]. Angular velocity levels in the animals that were euthanized following injury (average: $191.67 \pm 9.6 \mathrm{rad} / \mathrm{s}$ ) did not differ from those in animals that survived (average: $193.8 \pm 6.7$ $\mathrm{rad} / \mathrm{s})$.

\section{Animals Excluded from Analysis}

Two injured animals were excluded from the behavioral analysis. One animal from the Inj + Fol group (velocity: $185 \mathrm{rad} / \mathrm{s}$ ) had apnea following injury, was hypermetric and received steroids. On pathologic examination, this animal had some axonal injury in the deep white matter of the frontal and temporal lobes and brain stem ischemia. One more animal from the Inj + Fol group (velocity: $190 \mathrm{rad} / \mathrm{s}$ ) became weak on day 3 following injury and required hand feeding and was not tested on day 4 . On pathologic examination, this animal had extensive subarachnoid and intraparenchymal hemorrhage with extensive infarction. One uninjured animal from one of the groups that received meloxicam was not included in the behavioral analysis as its injured siblings were sacrificed following injury. The final group numbers used for the analysis were 7 animals in the Inj + Fol group, 8 animals in the Inj + Sal group, 8 animals in the Uninj + Fol group, and 7 animals in the Uninj + Sal group (fig. 3). Angular velocities in the Inj + Fol group (193.29 \pm 5.31 $\mathrm{rad} / \mathrm{s}$ ) did not differ from those in the Inj + Sal group $(194.25 \pm 8.11 \mathrm{rad} / \mathrm{s} ; \mathrm{p}=0.79)$ (table 1$)$.

Folic Acid and Early Functional Recovery

\section{Loss of Consciousness}

The duration of unconsciousness did not differ between injured groups (Inj + Fol: $6.32 \pm 0.12 \mathrm{~min}$; Inj + Sal: $6.23 \pm 0.09 \mathrm{~min} ; \mathrm{p}=0.9$ ). The duration was longer in uninjured animals that received folic acid compared to uninjured animals that received saline (Uninj + Fol: 4.55 $\pm 0.1 \mathrm{~min}$; Uninj + Sal: $2.53 \pm 0.12 \mathrm{~min} ; \mathrm{p}=0.01)$. The duration of unconsciousness was significantly longer in injured animals (6.27 $\pm 0.1 \mathrm{~min})$ compared to uninjured animals $(3.58 \pm 0.1 \mathrm{~min} ; \mathrm{p}=0.01)$ (table 1$)$.

\section{Folic Acid and Homocysteine Levels}

Folic acid levels did not differ between groups at preinjury baseline (Inj + Fol: $22.7 \pm 1.8 \mathrm{ng} / \mathrm{ml}$; Inj + Sal: 19.9 $\pm 4.23 \mathrm{ng} / \mathrm{ml}$; Uninj + Fol: $22.51 \pm 3.76 \mathrm{ng} / \mathrm{ml}$; Uninj + Sal: $19.72 \pm 5.56 \mathrm{ng} / \mathrm{ml} ; \mathrm{p}=0.45)$. However, levels of folic acid were significantly higher in the animals that received folic acid supplementation on day 6 compared to those that did not (Inj + Fol: $21.76 \pm 3.5 \mathrm{ng} / \mathrm{ml}$; Inj + Sal: 18.68 $\pm 4.75 \mathrm{ng} / \mathrm{ml}$; Uninj + Fol: $24 \pm 0 \mathrm{ng} / \mathrm{ml}$; Uninj + Sal: $18.08 \pm 4.84 \mathrm{ng} / \mathrm{ml} ; \mathrm{p}=0.005)$. Homocysteine levels did not differ at baseline between groups (Inj + Fol: $6.73 \pm$ $0.9 \mu \mathrm{mol} / \mathrm{l} ; \mathrm{Inj}+$ Sal: $6.88 \pm 1.72 \mu \mathrm{mol} / \mathrm{l}$; Uninj + Fol: $6.31 \pm 2.57 \mu \mathrm{mol} / \mathrm{l} ;$ Uninj + Sal: $5.45 \pm 1.86 \mu \mathrm{mol} / \mathrm{l} ; \mathrm{p}=$ 0.57 ) and were not significantly lower at day 6 in animals that received supplementation compared to those that did not (Inj + Fol: $7.13 \pm 2.48 \mu \mathrm{mol} / \mathrm{l} ;$ Inj + Sal: $7.06 \pm 3.09$ $\mu \mathrm{mol} / \mathrm{l}$; Uninj + Fol: $5.33 \pm 2.46 \mu \mathrm{mol} / \mathrm{l}$; Uninj + Sal: $7 \pm 1.68 \mu \mathrm{mol} / \mathrm{l} ; \mathrm{p}=0.45)($ table 1$)$.

\section{Body Weights}

Body weights at preinjury baseline did not differ between groups (Inj + Fol: $2.24 \pm 0.52 \mathrm{~kg}$; Inj + Sal: $2.01 \pm$ $0.24 \mathrm{~kg}$; Uninj + Fol: $1.8 \pm 0.54 \mathrm{~kg}$; Uninj + Sal: $1.99 \pm$ 
Fig. 4. Representative pathology from injured (a-c) and uninjured (d-f) animals. $\mathbf{a}$, d Slices from the frontal lobe of the piglet. Note white matter injury (circled area) in the deep white matter of the injured animal. b, e Slices from the parietal lobe, demonstrating extensive deep white matter injury in the injured animal with a small amount of white matter injury in the uninjured animal. c, f Brain stem with areas of white matter injury and infarction (shaded area) in the injured animal.
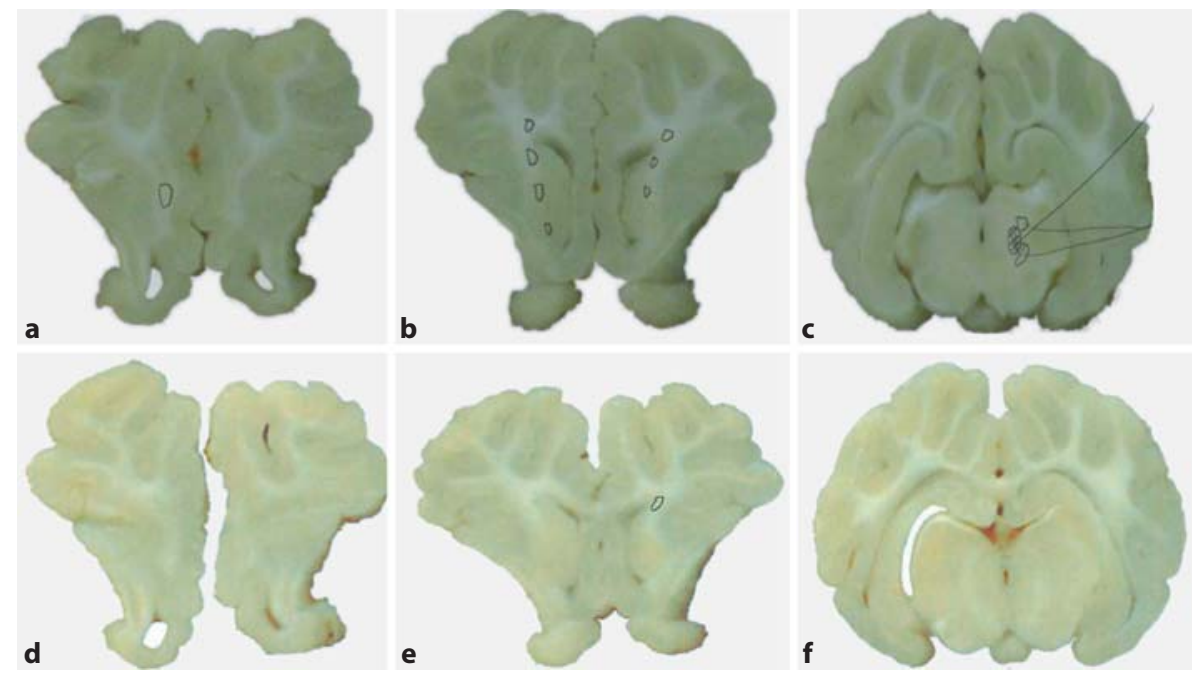

$0.39 \mathrm{~kg} ; \mathrm{p}=0.31$ ) nor was there a difference on day 6 (Inj + Fol: $3.12 \pm 0.87 \mathrm{~kg}$; Inj + Sal: $2.95 \pm 0.39 \mathrm{~kg}$; Uninj + Fol: $2.58 \pm 0.79 \mathrm{~kg}$; Uninj + Sal: $2.47 \pm 0.78 \mathrm{~kg} ; \mathrm{p}=0.44$ ). There was no difference in weight gain in injured treated compared to untreated animals, between injured and uninjured animals $(\mathrm{p}=0.12)$, or between treated and untreated animals $(\mathrm{p}=0.35)$ (table 1$)$.

\section{Pathology}

The primary focus of the pathology analysis was to evaluate the neuroprotective effect of folic acid treatment in the brains of injured animals. In addition, 4 uninjured brains were analyzed to evaluate the effect of a microdialysis probe that was being used on a trial basis. A total of 19 brains were included in the analysis (Inj + Fol: $\mathrm{n}=$ $7 ;$ Inj + Sal: $\mathrm{n}=8$; Uninj + Sal: $\mathrm{n}=4$ ). The majority of white matter injury was seen in the deep white matter of the frontal lobes, with some piglets demonstrating deep white matter injury in the parietal and temporal lobes. Axonal injury was not significantly different in the injured animal groups (Inj + Fol: $0.25 \%$ of total brain; Inj + Sal: $0.18 \%$ of total brain; $\mathrm{p}=0.2$ ), but it was significantly greater than in uninjured animals (Uninj + Sal: 0.003\%; $\mathrm{p}=0.003)$.

All animals had ischemic injury. In the injured groups, ischemic injury was also seen mainly in the frontal lobe, with a few animals having areas of ischemia in the parietal lobes. Four animals had areas of ischemia in the brain stem, and 1 of these 4 animals had apnea following injury (fig. 4). In addition, in the Uninj + Sal animals, ischemic injury was limited to the area of the microdialysis probe used on day 6 prior to sacrifice. However, there was no significant difference in infarct size across groups (Inj + Fol: 0.47\%; Inj + Sal: 0.09\%; Uninj + Sal: 0.16\%; p = 0.34).

\section{Neurobehavioral Assessments}

\section{Open-Field Testing}

On day 1, injured animals explored the test space by entering more zones of the test space per minute epoch than the uninjured animals (fig. 5). In subgroup analysis, Inj + Fol animals explored the test space more than all other groups (fig. 6); this difference was not seen on day 4. In analysis of common open-field behaviors (sniffing floors, sniffing walls, sniffing toys, walking, standing still, pushing toys and running), injured animals ran fewer minute-long epochs compared to uninjured animals (fig. 7). In subgroup analysis, Inj + Sal animals spent a significantly greater percentage of time laying down on day 1 , averaging $5 \%$ of the 10 -min-long intervals, compared to the other 3 groups, who did not lie down at all ( $0 \%$ of 10 -min-long intervals) (fig. 8 ). There was no difference between treated and untreated animals on day 1 , and no significant difference between injured and uninjured animals, treated and untreated animals and subgroups on day 4 following injury.

During the 10-min interval of interaction with Pig-X (the uninjured con stimulus), uninjured animals were in closer proximity to Pig-X compared to injured animals on both days 1 and 4 following injury (fig. 9). There was no treatment effect or subgroup difference in proximity to Pig-X on either day of testing, and no difference in range of behaviors with Pig-X. 
5

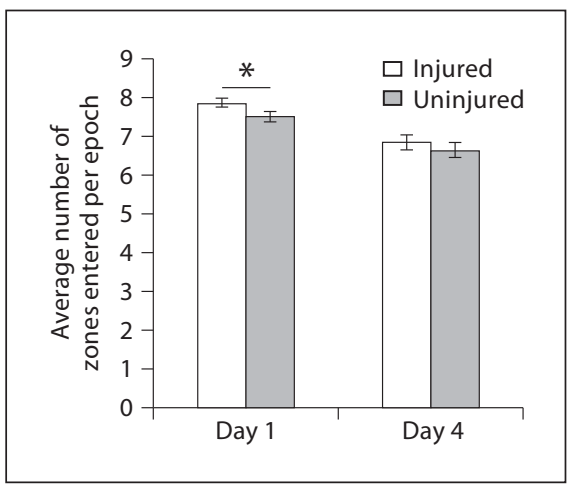

6

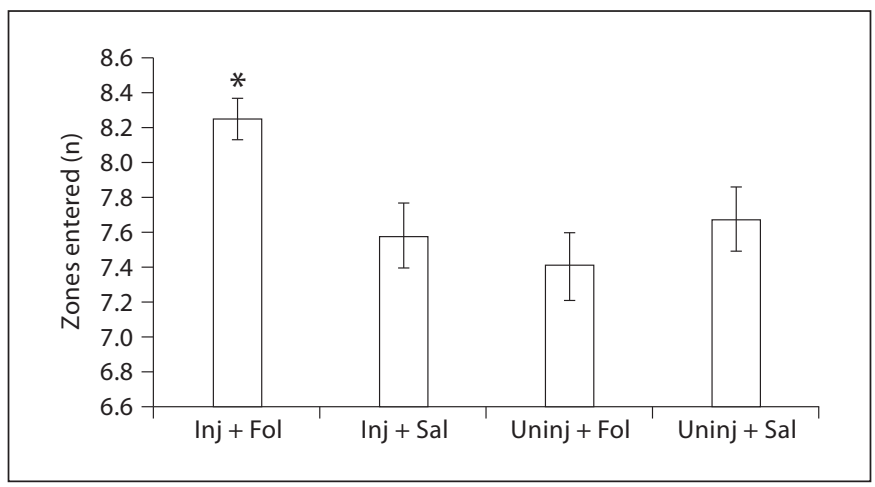

Fig. 5. Zones entered in open-field testing. On day 1, injured animals entered more zones compared to uninjured animals during the $10 \mathrm{~min}$ of open-field testing. This difference was not seen on day 4 of testing. ${ }^{*} \mathrm{p}<0.05$. Fig. 6. Zones entered in open-field testing. On day 1 of testing, injured animals that received folic acid entered significantly more zones than all other groups. ${ }^{*} \mathrm{p}<0.05$.

7

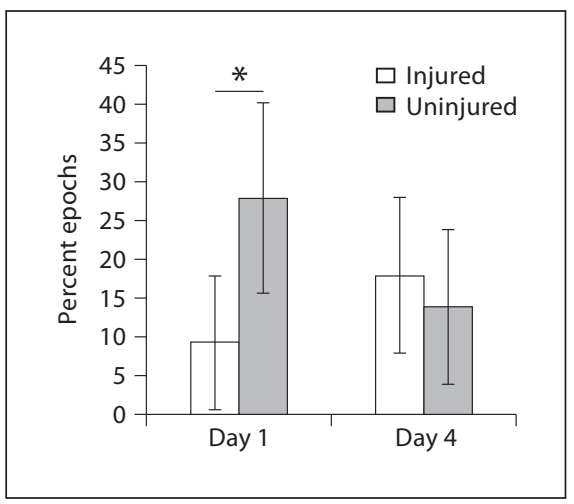

8

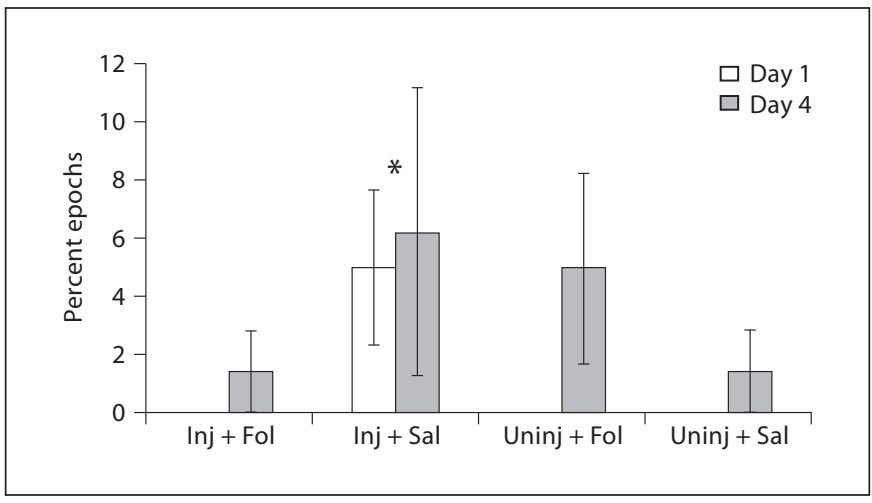

Fig. 7. Open-field testing behaviors. Uninjured animals ran significantly more on day 1 of open-field testing compared to injured animals. This difference was not seen on day $4 .{ }^{*} \mathrm{p}<0.05$.

Fig. 8. Open-field testing behaviors. Inj + Sal animals spent significantly more time laying down on day 1 of open-field testing, indicating less interest in exploring the test space, compared to the other groups, who did not lie down at all during the first day of testing. All animals spent time laying down on day 4 of testing, indicating habituation to the test space; however, there were no significant differences between groups. ${ }^{*} \mathrm{p}<0.05$.

\section{Mirror Test}

All groups spent significantly more time in front of the mirror compared to the reversed mirror and null zones on both days 1 and 4, and significantly more time in front of the mirror on day 1 compared to day 4 (fig. 10). Snout contact with the mirror was a relatively common behavior on day 1 ( $66 \pm 23.13 \%$ intervals), but was observed less frequently on day 4 (44 $\pm 23.75 \%$ intervals). Interestingly, all animals spent significantly more time in front of the mirror in the first minute of the evaluation compared to the last minute on day 1 (1st min: $13.49 \pm$ $10.75 \mathrm{~s} ; 10$ th min: $7.13 \pm 6.45 \mathrm{~s}$ ) and day 4 (1st min: 5.89 $\pm 6.27 \mathrm{~s} ; 10$ th min: $2.21 \pm 3.39 \mathrm{~s}$ ). Injured animals spent more time in front of the mirror on both days 1 and 4 compared to uninjured animals (fig. 11); however, there were no differences between treated and untreated animals and between subgroups on either testing day in time spent in front of the mirror or in specific mirror behaviors. 
Fig. 9. Socialization with male sibling (Pig$\mathrm{X})$. Injured animals were further away from Pig-X compared to uninjured animals during the period of socialization testing on days 1 and 4 of testing. ${ }^{*} \mathrm{p}<0.05$. Fig. 10. Mirror test. All animals spent more time in front of the mirror compared to the reversed mirror and null zone on days 1 and 4 . In addition, all animals spent more time in front of the mirror on day 1 compared to day 4, indicating habituation to the mirror over time. ${ }^{\dagger} \mathrm{p}<0.05 \mathrm{com}-$ pared to reversed mirror and null zone; ${ }^{*} \mathrm{p}<0.05$ between day 1 and day 4 .

Fig. 11. Mirror test. Injured animals spent more time per minute epoch in front of the mirror compared to uninjured animals on both days 1 and $4 .{ }^{*} \mathrm{p}<0.05$.

Fig. 12. Glass barrier testing. Animals that received treatment with folic acid made less errors per trial on day 4 compared to untreated animals. This difference was not seen on day 1 of testing. ${ }^{*} \mathrm{p}<0.05$.

Fig. 13. Food cover testing. Injured animals made more errors per trial on day 4 compared to uninjured animals. This difference was not seen on day 1 of testing. ${ }^{*} \mathrm{p}<0.05$.

Fig. 14. Beam testing. Treated animals completed the beam test quicker than the untreated animals on day 1 . This difference was not seen on day $4 .{ }^{*} \mathrm{p}<0.05$.
9

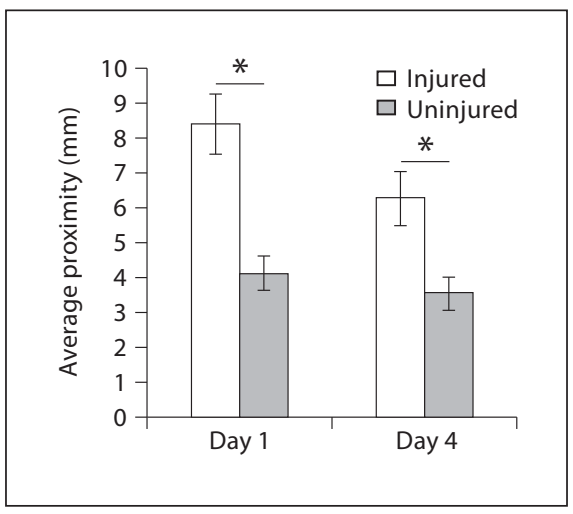

10

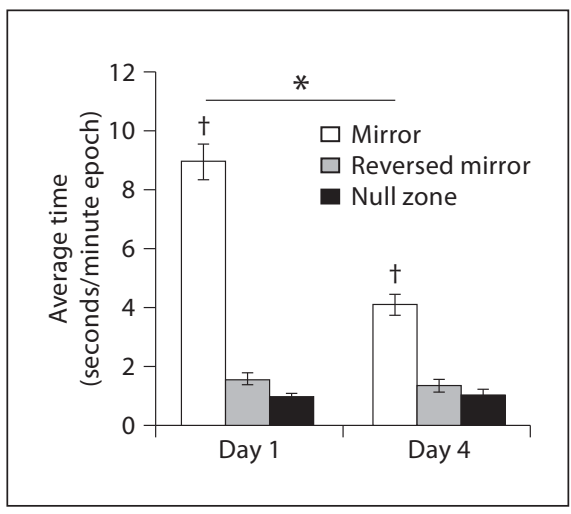

11

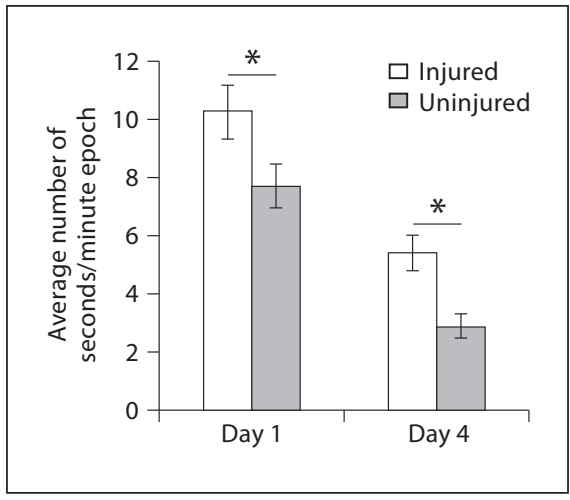

12

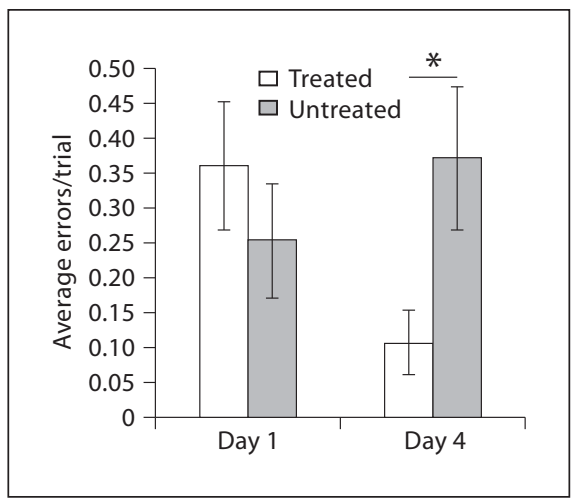

14

13

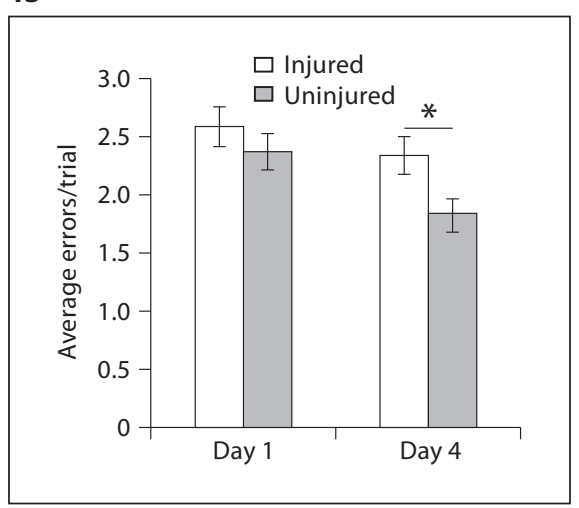

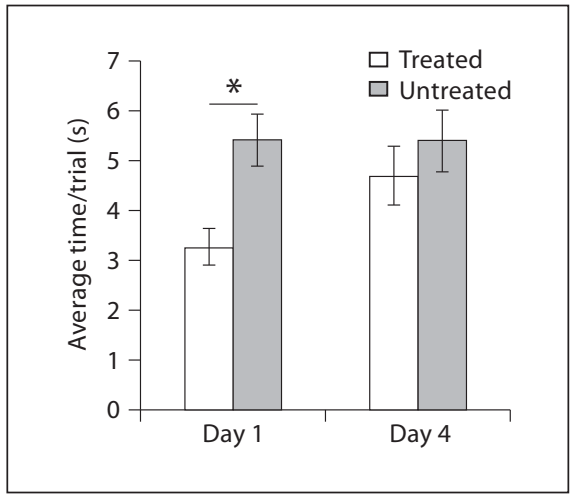

\section{Glass Barrier Task}

Latencies to reach the food reward did not vary across groups on day 1 (average: $6.66 \pm 3.43 \mathrm{~s} /$ trial) or day 4 (average: $5.33 \pm 4.28 \mathrm{~s} /$ trial). All groups committed the same number of errors per successive trial on day 1 (average: $0.4 \pm 0.74$ errors/trial). On day 4 , animals that re- ceived treatment with folic acid made fewer errors than untreated animals (fig. 12); however, there was no difference between injured and uninjured animals. There was no difference between groups in glass barrier failure rates on day 1 (average: $0.76 \pm 1.28$ failures/day) and day 4 (average: $0.41 \pm 0.82$ failures/day). All animals showed a 


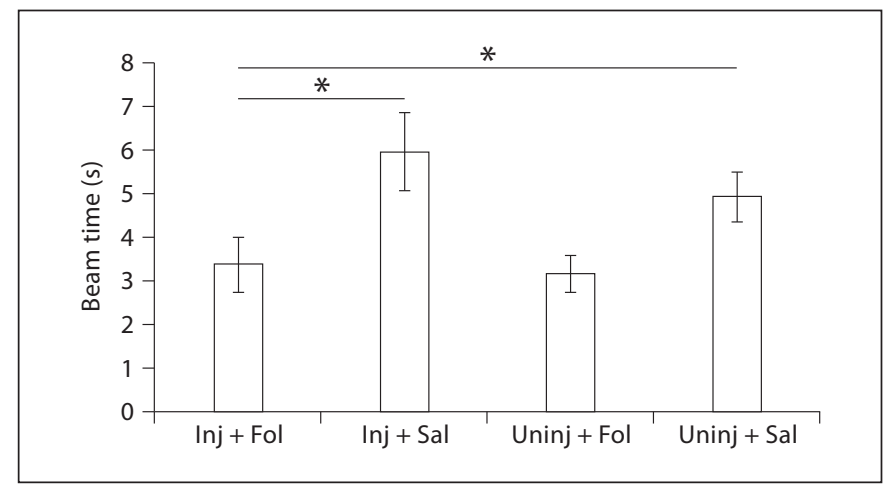

Fig. 15. Beam testing. On day 1 of testing, Inj + Fol animals had significantly shorter beam completion times compared to Inj + Sal and Uninj + Sal animals. ${ }^{*} \mathrm{p}<0.05$.

learning effect, having shorter latencies to reach the food reward, fewer errors and failures on day 4 compared to day 1.

\section{Food Cover Task}

There was no difference in latency to reach the food reward, errors or failure rate on day 1 of testing between injured and uninjured animals, treated and untreated animals, and subgroups. Although Inj + Fol animals showed a shorter latency to reach the food reward compared to other groups on day 1 (Inj + Fol: $10.1 \pm 5.66 \mathrm{~s}$; Inj + Sal: $12 \pm 5.65 \mathrm{~s}$; Uninj + Fol: $12.07 \pm 3.85 \mathrm{~s}$; Uninj + Sal: 15.5 $\pm 3.96 \mathrm{~s})$ and day 4 (Inj + Fol: $7.2 \pm 4.21 \mathrm{~s} ; \mathrm{Inj}+$ Sal: 8.83 $\pm 3.21 \mathrm{~s}$; Uninj + Fol: $9.23 \pm 4.07 \mathrm{~s}$; Uninj + Sal: $9.25 \pm$ $5.41 \mathrm{~s})$, this did not reach statistical significance. All groups committed the same number of errors per successive trial on day 1 (average: $2.47 \pm 1.48$ errors/trial). On day 4 of testing, injured animals made more errors than uninjured animals (fig. 13); however, there was no effect of treatment. There was no difference between groups in food cover failure rates on day 1 (average: $4.76 \pm 1.46$ failures/day) and day 4 (average: $4.59 \pm 2.21$ failures/ day).

\section{Balance Beam}

On day 1, animals that received treatment with folic acid had shorter latencies to complete the balance beam task compared to untreated animals (fig. 14). Inj + Fol animals had significantly lower latencies to reach the food reward compared to the Inj + Sal and Uninj + Sal animals (average: $3.38 \pm 2.91 \mathrm{~s} /$ trial vs. $5.9 \pm 4.71 \mathrm{~s} /$ trial and $4.93 \pm 3.23 \mathrm{~s} /$ trial) (fig. 15). This effect was not seen on day 4 . There were no significant differences in beam

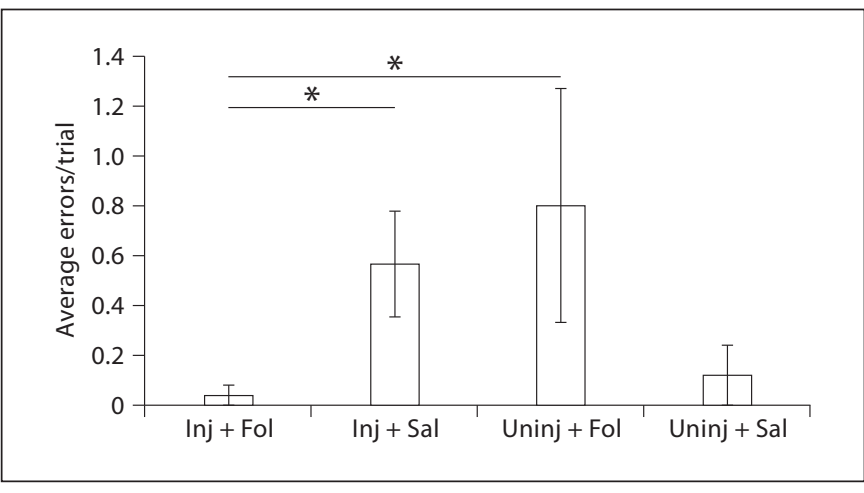

Fig. 16. T-maze testing (normal trials). On day 1 of testing, Inj + Fol animals made significantly less errors on normal trials than Inj + Sal and Uninj + Fol animals. ${ }^{*} \mathrm{p}<0.05$.

errors or failure rates between groups on either testing day. All animals showed a learning effect, having shorter latencies to reach the food reward, fewer errors and failures on day 4 compared to day 1 .

\section{T-Maze Testing}

On both days, Inj + Fol animals had a higher training pass percentage compared to other groups; however, this was not statistically significant on day 1 (average: 64.21 $\pm 28.74 \%$ ) or day 4 (average: $55.28 \pm 43.18 \%$ ). On day 1 , Inj + Fol animals and Uninj + Sal animals had significantly shorter times to reach the food reward on normal trials compared to the Inj + Sal and Uninj + Fol animals $(4.12 \pm 2.12$ and $3.6 \pm 2.16 \mathrm{~s} /$ trial, compared to $5.07 \pm$ 2.5 and $7.3 \pm 17$ s/trial). In addition, Inj + Fol and Uninj + Sal animals made significantly fewer errors during these trials compared to Inj + Sal and Uninj + Fol animals (fig. 16), but these group differences were not seen on day 4 . There were no differences in intramaze change or reversal trial assessments. There were no significant differences between injured and uninjured animals and between treated and untreated animals on either testing days. All animals showed a learning effect, having shorter latencies to reach the food reward, fewer errors and failures on day 4 compared to day 1 .

\section{CCD Score}

The CCD score is a measure of the overall neurobehavioral performance of a piglet, based on performance on tests where there are the most consistent responses among shams. Five measures are included in the calculation of this score: T-maze training failure rate; T-maze intramaze change time in contact with a novel object; la- 


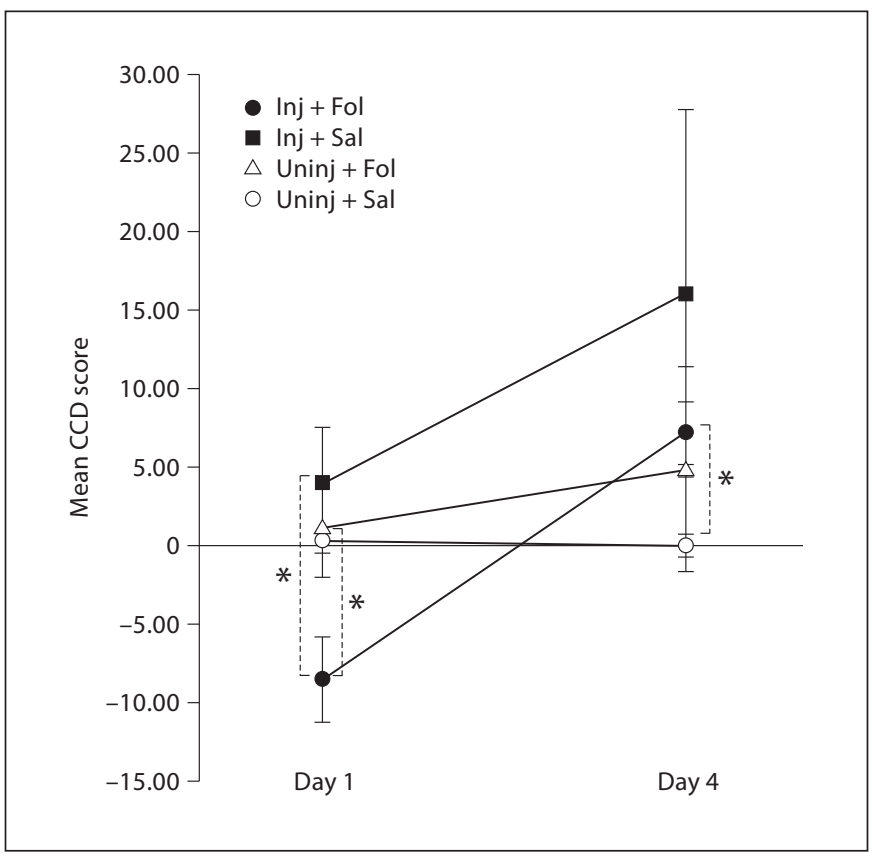

Fig. 17. CCD. On day 1 following injury, Inj + Fol animals had a significantly lower CCD score compared to Inj + Sal and uninjured animals (Uninj + Fol, Uninj + Sal). On day 4, although Inj + Fol animals had a lower CCD score compared to Inj + Sal animals, this was not statistically significant, but was significantly higher than that of uninjured animals. Error bars = standard error. ${ }^{*} \mathrm{p}<0.05$.

tency to food reward for T-maze normal trials and Tmaze reversal trials, and sniffing the walls on open-field testing [19]. On day 1 following injury, Inj + Fol animals had a significantly lower CCD score compared to Inj + Sal $(\mathrm{p}=0.02)$ and uninjured animals $(\mathrm{p}<0.05)$. On day 4 , although Inj + Fol animals had a lower CCD score than Inj + Sal animals, this was not statistically significant, but was significantly higher than that in Uninj + Sal animals $(p=0.02)$ (fig. 17). CCD did not correlate well with percent white matter injured, with a correlation coefficient of 0.01 on day 1 and 0.03 on day 4 following injury.

\section{Discussion}

In this novel study of interventions for pediatric TBI, we used an immature swine model of diffuse brain injury in the child to assess functional improvements. There are numerous studies in the literature assessing behavioral testing following rodent TBI. Most of these studies involve the Morris water maze test [21], and include numerous intervention studies of rodent TBI [22-26]. However, the piglet offers a better model of the human brain, with similar cortical grey-white differentiation, gyral pattern and physiologic responses to TBI in humans $[27,28]$. Outcomes assessed in previous swine TBI intervention studies have included mean arterial pressure [29-31], intracranial pressure [30-32], cerebral blood flow [29-32], brain tissue oxygen tension [29], cerebral metabolic rate of oxygen [31], immunohistochemistry [29-31], histopathology [30, 31, 33] and lesion volume by magnetic resonance imaging $[29,32]$. Simple functional testing has been performed in swine TBI studies [32,34] with the use of a veterinary coma scale, similar to the Glasgow Coma Scale [35] used in humans. A cumulative score is calculated from individual motor function, eye function and respirations; however, this testing does not assess multiple domains of animal behavior. In summary, this is the first study to describe the application of complex functional testing to assess an intervention outcome in a swine model of TBI.

In our first paper on neurobehavioral testing, we reported that injured animals had less interest in exploring their surroundings compared to uninjured animals [18]. In this study, we observed that injured animals entered more zones and explored the test space more during the period of open-field testing; however, the injured animals were observed to run less than the uninjured animals on day 1 of testing, and interestingly, injured animals that received saline spent more time laying down during open-field testing, suggesting that this subgroup is similar to our previous injured animals with a decreased interest in the novel test space [18]. Of note, all animals spent time laying down on day 4 of testing, indicating habituation to the test space. Injured animals were also observed to be further away from their male sibling (Pig$\mathrm{X}$ ) during both days of behavioral testing, indicating that injury impaired socialization behavior; however, unlike in our previous study, injured piglets did not receive less head butting from Pig-X compared to uninjured animals. Injured animals made more food cover errors on day 4 following injury compared to uninjured animals, indicating deficits in primary olfactory function were present in injured animals as described in our previous paper. Furthermore, similar to our first paper, all animals showed a learning effect, making fewer errors and having shorter latencies and fewer failures on day 4 of testing compared to day 1 on glass barrier, balance beam and T-maze testing.

We report significant improvements in functional outcome on day 1 of testing for Inj + Fol animals com- 
pared to other groups in open-field exploration and CCD score. In addition, on day 1 , Inj + Fol animals were significantly quicker in completing the balance beam task than the Inj + Sal and Uninj + Sal animals. This finding suggests some early improvement in motor function following injury in the animals that received folic acid supplementation. The Inj + Fol and Uninj + Sal animals performed better than Inj + Sal and Uninj + Fol animals on T-maze testing on day 1. Interestingly, injured animals did not spend more time in contact with the novel object than in the previous repetitive injury study [19]. All the significant behaviors between subgroups that were observed on day 1 following injury were not observed on day 4 of behavioral testing. We conclude that folic acid improves early functional outcome following TBI.

Animals that received folic acid supplementation (Inj + Fol and Uninj + Fol) also had shorter latencies to complete the beam task on day 1 of testing, and made fewer errors on glass barrier testing on day 4 following injury, compared to untreated animals (Inj + Sal and Uninj + Sal), indicating better motor function and visual problem solving in animals that received folic acid supplementation. Interestingly, there was no difference between the Inj + Fol and Uninj + Fol animals on beam testing. However, we hesitate to conclude that animals that received saline did worse than animals that received folic acid supplementation as there is a lot of variability in the behaviors observed in the injured and uninjured animals. We are currently working on refining our behavioral assessments to find the most sensitive measures to detect differences between injured and uninjured animals.

During T-maze normal trials on day 1, uninjured animals that received folic acid supplementation made more errors compared to the Inj + Fol and Inj + Sal animals. Because our study design precludes distinguishing between contributions of animal-to-animal variability and group-to-group variability to the overall outcome range, future behavioral assessments should include a preliminary, preinjury baseline test day to allow us to normalize each subject's findings to his or her own baseline performance.

Folic acid supplementation did not significantly reduce injured brain volume. The amount of white matter axonal injury was similar to that seen in our previous studies, with a 5-day postinjury survival (0.25\%) [19]. Thus, despite improvements in early neurological function, folic acid treatment for 6 days did not significantly reduce injured brain volume. In the rodent model of spinal cord injury, folic acid supplementation for 2 weeks enhanced neurological recovery from a spinal cord con-

Folic Acid and Early Functional Recovery tusion [14], suggesting that a longer duration of treatment may be more beneficial following central nervous system injury. Folic acid decreases NMDA-mediated neuronal cell death in a dose- and time-dependent manner [16]. The dose chosen in this study was the dose that had been shown to enhance repair processes in an adult rat model of spinal cord injury [14], and was equivalent to the dose used on prevention of neural tube defects in pregnant women with a previous neural tube defect pregnancy [7]. In this study, we demonstrated that we were able to increase serum levels of folic acid significantly by folic acid IP supplementation; however, we speculate whether a higher dose of folic acid may have been more beneficial. In addition, other measures such as those of NMDA-mediated apoptotic cell death including TUNEL staining may be investigated in future studies as histopathologic markers of outcome [36, 37].

Folic acid levels were significantly higher in the animals that received folic acid supplementation compared to untreated animals, demonstrating we were able to increase serum levels with treatment; however, there were no significant reductions in plasma homocysteine levels in animals that received folic acid supplementation, which is the hypothesized mechanism of action of the protective effect of folic acid [15]. There is a transport system for folic acid across the blood-brain barrier [38], but we did not assess cerebral concentrations of folic acid to see if folic acid was entering the injured brain across the blood-brain barrier, or to see if there was a reduction in cerebral homocysteine levels that could have been accomplished by microdialysis.

In this communication, we introduce a new assessment using a mirror and another novel object (reversed mirror), and compare piglet interactions with a control wall segment with no alterations (null zone). All animals spent more time in front of the mirror compared to the reversed mirror and null zone. These findings complement those from a recent study by Broom et al. [39], where 4- to 8-week-old piglets spent time in front of the mirror and were able to use information seen in the mirror to find food around a corner. The ability to recognize self when looking in a mirror has been shown in other species including primates and occurs in humans at 9 months of age [40]. While it is not clear whether piglets think they are seeing another piglet or have 'self-awareness', the piglets demonstrated significantly more interest in interacting with the mirror than with another novel object. In our current study, the piglets' interest in the mirror declined over the 10 -min period, and on the subsequent testing day, which is consistent with the observation by 
Broom et al. [39], habituation to the mirror occurred over time. Interestingly, injured piglets spent significantly more time in front of the mirror on both testing days compared to uninjured piglets.

The overall mortality rate following TBI in this study is similar to that reported in our previous multiday survival TBI studies $[18,19]$, with hard palate fractures and inability to feed leading to sacrifice of an animal following injury. In our first 2 study groups, 3 animals never regained consciousness following injury and had large subdural hematomas on necropsy that were attributed the use of the new preinjury analgesic meloxicam. Meloxicam is a nonsteroidal antiinflammatory agent, and it potentially caused these findings by its antiplatelet effect. Although meloxicam is known to prolong bleeding time in other animal models [20], this is the first report of severe effects associated with the use of this medication. We saw no further mortalities related to injury in the last 8 groups, where we used buprenorphine as an analgesic.
In conclusion, we report a novel study describing the application of complex functional testing to assess an intervention outcome in a swine model of TBI. Our results suggest that folic acid supplementation may enhance early functional recovery following TBI in a piglet model of TBI. Future work in this area will focus on potential mechanisms of the beneficial effect of folic acid, longterm neurological outcome, longer treatment durations and optimal dosage.

\section{Acknowledgments}

We are grateful to Ashley Young for her technical help in behavioral testing. This study was supported by the Thrasher esearch Foundation New Investigator award (M.Y.N.), the Endowed Chair in Critical Care Medicine from the Children's Hospital of Philadelphia (M.A.H.) and National Institutes of Health grant R01 NS39679 (S.S.M.).

\section{References}

1 Langlois JA, Rutland-Brown W, Thomas KE: The incidence of traumatic brain injury among children in the United States: differences by race. J Head Trauma Rehabil 2005; 20:229-238.

-2 Kochanek PM, Clark RS, Ruppel RA, Adelson PD, Bell MJ, Whalen MJ, Robertson CL, Satchell MA, Seidberg NA, Marion DW, Jenkins LW: Biochemical, cellular, and molecular mechanisms in the evolution of secondary damage after severe traumatic brain injury in infants and children: lessons learned from the bedside. Pediatr Crit Care Med 2000;1:4-19.

>3 Graham DI, Ford I, Adams JH, Doyle D, Lawrence AE, McLellan DR, Ng HK: Fatal head injury in children. J Clin Pathol 1989; 42:18-22.

4 Shannon P, Smith CR, Deck J, Ang LC, Ho $M$, Becker L: Axonal injury and the neuropathology of shaken baby syndrome. Acta Neuropathol 1998;95:625-631.

5 Povlishock JT, Pettus EH: Traumatically induced axonal damage: evidence for enduring changes in axolemmal permeability with associated cytoskeletal damage. Acta Neurochir Suppl 1996;66:81-86.

6 Smithells RW, Sheppard S, Schorah CJ: Vitamin deficiencies and neural tube defects. Arch Dis Child 1976;51:944-950.
7 Czeizel AE, Dudás I: Prevention of the first occurrence of neural-tube defects by periconceptional vitamin supplementation. $\mathrm{N}$ Engl J Med 1992;327:1832-1835.

8 Reynolds EH: Benefits and risks of folic acid to the nervous system. J Neurol Neurosurg Psychiatry 2002;72:567-571.

$\checkmark 9$ Ho PI, Ashline D, Dhitavat S, Ortiz D, Collins SC, Shea TB, Rogers E: Folate deprivation induces neurodegeneration: roles of oxidative stress and increased homocysteine. Neurobiol Dis 2003;14:32-42.

10 Reynolds EH: Folic acid, aging depression and dementia. BMJ 2002;324:1512-1515.

- 11 Endres M, Ahmadi M, Kruman I, Biniszkiewicz D, Meisel A, Gertz K: Folate deficiency increases postischemic brain injury. Stroke 2005;36:321-325.

12 Martin H, Lindblad B, Norman M: Endothelial function in newborn infants is related to folate levels and birth weight. Pediatrics 2007;119:1152-1158.

13 van Guelpen B, Hultdin J, Johansson I, Stegmayr B, Hallmans G, Nilsson TK, Weinehall L, Witthöft C, Palmqvist R, Winkvist A: Folate, vitamin $B_{12}$, and risk of ischemic and hemorrhagic stroke: a prospective, nested case-referent study of plasma concentrations and dietary intake. Stroke 2005;36:14261431.

- 14 Iskandar MJ, Nelson A, Resnick D, Skene JH, Gao P, Johnson C, Cook TD, Hariharan N: Folic acid supplementation enhances repair of the adult central nervous system. Ann Neurol 2004;56:221-227.
15 Mattson MP, Shea TB: Folate and homocysteine metabolism in neural plasticity and neuroregenerative disorders. Trends Neurosci 2003;26:137-146.

16 Lin Y, Desbois A, Jiang S, Hou ST: Group B vitamins protect murine cerebellar granule cells from glutamate/NMDA toxicity. Neuroreport 2004; 15:2241-2244.

17 Raghupathi R, Margulies SS: Traumatic axonal injury after closed head injury in the neonatal pig. J Neurotrauma 2002;19:843-853.

18 Friess SH, Ichord R, Owens K, Ralston J, Rizol R, Overall KL, Smith C, Helfaer MA, Margulies SS: Neurobehavioral functional deficits following closed head injury in the neonatal pig. Exp Neurol 2007;204:234-243.

-19 Friess SH, Ichord RN, Ralston J, Ryall K, Helfaer MA, Smith C, Margulies SS: Repeated traumatic brain injury affects composite cognitive function in piglets. J Neurotrauma 2009;26:1111-1121.

20 Luna SP, Basílo AC, Steagall PV, Machado LP, Moutinho FQ, Takahira RK, Brandão $\mathrm{CV}$ : Evaluation of adverse effects of longterm oral administration of carprofen, etodolac, flunixin meglumine, ketoprofen, and meloxicam in dogs. Am J Vet Res 2007;68: 258-264.

21 Morris R: Developments of a water-maze procedure for studying spatial learning in the rat. J Neurosci Methods 1984;11:47-60. 
22 Qu C, Lu D, Goussev A, Schallert T, Mah$\operatorname{mood}$ A, Chopp M: Effect of atorvastatin on spatial memory, neuronal survival, and vascular density in female rats after traumatic brain injury. J Neurosurg 2005;103:695-701.

23 Hoane MR, Akstulewicz SL, Toppen J: Treatment with vitamin $B_{3}$ improves functional recovery and reduces GFAP expression following traumatic brain injury in rats. J Neurotrauma 2003;20:1189-1199.

-24 Hoane MR, Wolyniak JG, Akstulewicz SL: Administration of riboflavin improves behavioral outcome and reduces edema formation and glial fibrillary acidic protein expression after traumatic brain injury. J Neurotrauma 2005;22:1112-1122.

25 Alessandri B, Rice AC, Levasseur J, DeFord M, Hamm RJ, Bullock MR: Cyclosporin A improves brain tissue oxygen consumption and learning/memory performance after lateral fluid percussion injury in rats. J Neurotrauma 2002;19:829-841.

-26 Lu D, Mahmood A, Qu C, Goussev A, Schallert T, Chopp M: Erythropoietin enhances neurogenesis and restores spatial memory in rats after traumatic brain injury. J Neurotrauma 2005;22:1011-1017.

$\checkmark 27$ Armstead WM, Kurth CD: Different cerebral hemodynamic responses following fluid percussion brain injury in the newborn and juvenile pig. J Neurotrauma 1994;11:487497.
28 Durham SR, Raghupathi R, Helfaer MA, Marwaha S, Duhaime AC: Age-related differences in acute physiologic response to focal traumatic brain injury in piglets. Pediatr Neurosurg 2000;33:76-82.

29 Rosenthal G, Morabito D, Cohen M, Roeytenberg A, Derugin N, Panter SS, Knudson MM, Manley G: Use of hemoglobin-based oxygen-carrying solution-201 to improve resuscitation parameters and prevent secondary brain injury in a swine model of traumatic brain injury and hemorrhage: laboratory investigation. J Neurosurg 2008; 108: 575-587.

30 Stern S, Rice J, Philbin N, McGwin G, Arnaud F, Johnson T, Flournoy WS, Ahlers S, Pearce LB, McCarron R, Freilich D: Resuscitation with the hemoglobin-based oxygen carrier, HBOC-201, in a swine model of severe uncontrolled hemorrhage and traumatic brain injury. Shock 2009;31:64-79.

- 31 Fritz HG, Walter M, Holzmayr M, Brodhun M, Patt S, Bauer R: A pig model with secondary increase of intracranial pressure after severe traumatic brain injury and temporary blood loss. J Neurotrauma 2005;22:807-821.

-32 King DR, Cohn SM, Proctor KG: Changes in intracranial pressure, coagulation and neurologic outcome after resuscitation from experimental traumatic brain injury with hetastarch. Surgery 2004;136:355-363.

33 Zhang J, Groff RF 4th, Chen XH, Browne KD, Huang J, Schwartz ED, Meaney DF, Johnson VE, Stein SC, Rojkjaer R, Smith DH: Hemostatic and neuroprotective effects of human recombinant activated factor VII therapy after traumatic brain injury in pigs. Exp Neurol 2008;210:645-655.
4 Glass TF, Fabian MJ, Schweitzer JB, Weinberg JA, Proctor KG: The impact of hypercarbia on the evolution of brain injury in a porcine model of traumatic brain injury and systemic hemorrhage. J Neurotrauma 2001; 18:57-71.

35 Teasdale G, Jennett B: Assessment of coma and impaired consciousness: a practical scale. Lancet 1974;ii:81-84.

36 Yee WM, Frim DM, Isacson O: Relationships between stress protein induction and NMDA-mediated neuronal death in entorhinal cortex. Exp Brain Res 1993;94:193-202.

37 Wang H, Yu SW, Koh DW, Lew J, Coombs C, Bowers W, Federoff HJ, Poirier GG, Dawson TM, Dawson VL: Apoptosis-inducing factor substitutes for caspase executioners in NMDA-triggered excitotoxic neuronal death. J Neurosci 2004;24:10963-10973.

-38 Wu D, Pardridge DM: Blood-brain barrier transport of reduced folic acid. Pharm Res 1999;16:415-419.

39 Broom DM, Sena H, Moynihan KL: Pigs learn what a mirror image represents and use it to obtain information. Anim Behav 2009; 79:1037-1041.

40 Rochat P, Striano T: Who's in the mirror? Self-other discrimination in specular images by four- and nine-month-old infants. Child Dev 2002;73:35-46. 